\title{
Video-assisted thoracoscopic surgery lingular segmentectomy with pericardial resection and reconstruction
}

\author{
Takuya Nagashima ${ }^{1}$, Hiroyuki Ito ${ }^{1}$, Joji Samejima ${ }^{1}$, Kenji Inafuku ${ }^{1}$, Haruhiko Nakayama ${ }^{1}$ Munetaka Masuda ${ }^{2}$ \\ ${ }^{1}$ Department of Thoracic Surgery, Kanagawa Cancer Center, Yokohama, Japan; ${ }^{2}$ Department of Surgery, Yokohama City University, Yokohama, Japan \\ Correspondence to: Takuya Nagashima, MD. Department of Thoracic Surgery, Kanagawa Cancer Center, 2-3-2, Nakao Asahi-ku, Yokohama 241-8515, \\ Japan. Email: naga16@kcch.jp.
}

\begin{abstract}
We describe a patient with a metastatic lung mass who underwent video-assisted thoracoscopic surgery (VATS) lingular segmentectomy with pericardial resection and reconstruction as minimally invasive surgery. There was a metastatic mass arising from cervical cancer in the lingular segment. Because the mass was obviously larger than the intercostal space, we made a subxiphoid incision besides usual ports for VATS segmentectomy to remove a specimen. Intraoperatively, we found pericardial invasion by the mass. The pericardium was safely resected with the use of a bipolar vessel sealing system. To reconstruct the pericardium under thoracoscopy, we devised two techniques. One was the use of a thread to measure the defect size. A stretched thread was placed on the defect to determine its length. By referring to the defect length, we could cut a covering material of proper size. The other technique was that the needle tip should not be pointed towards the heart to ensure that the pericardium can be sutured safely. These techniques allowed us to reconstruct the pericardium relatively easily and safely.
\end{abstract}

Keywords: Video-assisted thoracoscopic surgery (VATS); combined resection; pericardial reconstruction

Received: 18 September 2018; Accepted: 26 November 2018; Published: 30 December 2018.

doi: 10.21037 /jovs.2018.12.06

View this article at: http://dx.doi.org/10.21037/jovs.2018.12.06

\section{Introduction}

Thoracoscopic procedures used to perform general thoracic surgery, such as lobectomy or wedge resection, are widely used as minimally invasive procedures for the management of lung cancer. However, it seems that complex surgical procedures, such as combined resection, are rarely performed thoracoscopically.

Combined resection of a neighboring organ was performed in 1,408 patients with lung cancer in 2014 according to the annual report of the fapanese Association for Thoracic Surgery (1). The pericardium was the third most commonly resected organ $(n=143)$. Although the rate of thoracoscopic surgery was not specified, it was probably low owing to the reasons described below.

Because the lungs are surrounded by vital organs, combined resection of neighboring organs requires special attention even during thoracotomy. Moreover, reconstruction is sometimes necessary, and high technical skills are needed to perform the procedure thoracoscopically. In particular, combined pericardial resection and reconstruction are technically difficult to perform under thoracoscopy because handling needles near the heart is inevitable.

We describe a patient in whom pericardial resection and reconstruction could be performed relatively safely via a thoracoscopic approach by using improved methods for measuring the defect size and suturing the pericardium and covering material.

\section{Clinical summary}

The patient was a 36-year-old woman who had a past history of cervical cancer. Computed tomographic (CT) examinations obtained during follow-up revealed a $48-\mathrm{mm}$ mass shadow in the left lingular segment (Figure 1A). A bronchoscopic biopsy revealed squamous-cell cancer arising from cervical cancer. There was no other lesion on CT, positron emission tomography (PET) scans, or magnetic resonance imaging (MRI) examinations. We 

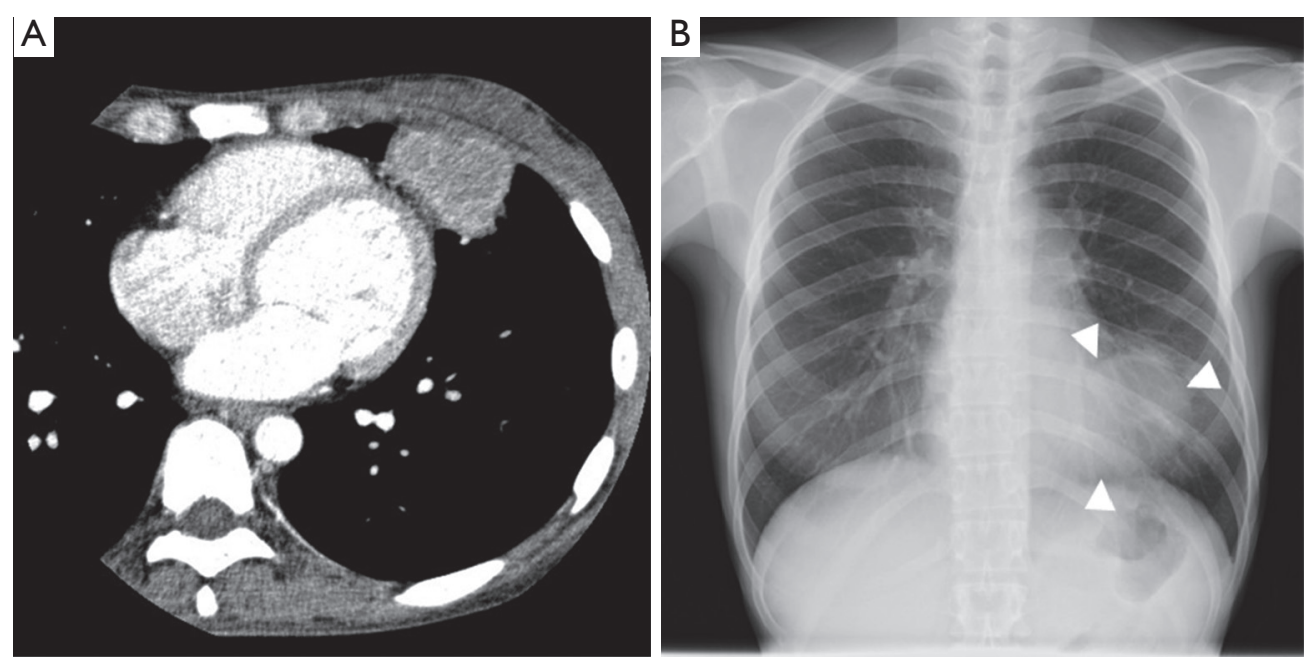

Figure 1 Preoperative image findings of the tumor. (A) A preoperative chest computed tomogram, showing a large mass adjacent to the pericardium; (B) a preoperative chest X-ray film, showing a mass larger than the intercostal space (arrowhead).

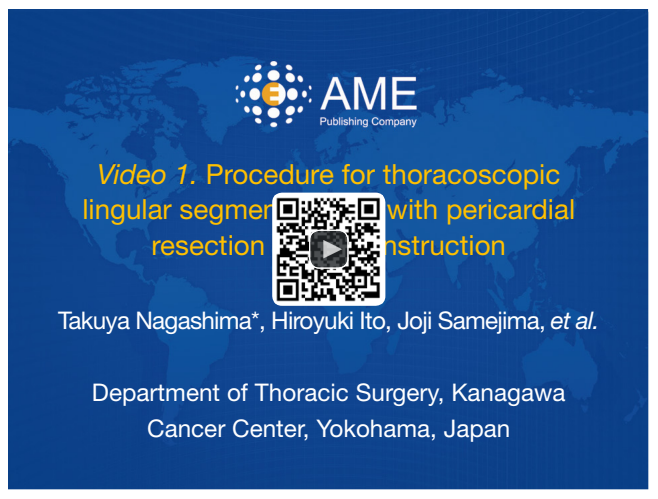

Figure 2 Procedure for thoracoscopic lingular segmentectomy with pericardial resection and reconstruction (2).

Available online: http://www.asvide.com/article/view/29142

performed thoracoscopic lingular segmentectomy with a $3-\mathrm{cm}$ subxiphoid incision to remove the specimen from the thoracic cavity in addition to using our usual ports for segmentectomy because the mass was obviously larger than the intercostal space according to the chest X-ray film (Figure 1B). During operation, we found pericardial invasion by the mass; pericardial resection and pericardial reconstruction with covering material were therefore concurrently performed. The operation time was $210 \mathrm{~min}$, and the blood loss was $50 \mathrm{~mL}$. The postoperative course was uneventful. On postoperative day 7 , the patient was discharged. There were no cardiovascular events as of 6 months postoperatively. Pathological examination revealed that the tumor measured $4.7 \mathrm{~cm} \times 4.4 \mathrm{~cm} \times 3.6 \mathrm{~cm}$ with pericardial invasion, no hilar lymph node metastasis, and a negative surgical margin.

\section{Operative techniques (Figure 2)}

After general anesthesia and intubation with a double-lumen endobronchial tube, the patient was placed in the supine position. A 3-cm skin incision was made in the subxiphoid area. The xiphoid process was removed, and the backside of sternum was dissected towards the left side of the thoracic cavity to make a tunnel for specimen removal. An Alexis ${ }^{\circledR}$ Wound Retractor XXS (Applied Medical, Rancho Santa Margarita, California, USA) was placed into this incision.

Then the posture was changed, and the patient was placed in the right lateral position. The operator stood on the dorsal side of the patient. A 2-cm skin incision was made along the sixth intercostal midaxillary line, and an Alexis ${ }^{\circledR}$ Wound Retractor XXS was placed into the incision to serve as an aperture for the operator's right hand. A 7-mm trocar was inserted into the fourth intercostal anterior axillary line to serve as an aperture for the operator's left hand. A $10-\mathrm{mm}$ trocar was inserted into the fifth intercostal posterior axillary line. A $10-\mathrm{mm}$ camera and an obliqueviewing endoscope (30 degrees) were used. A 15-mm skin incision was made along the seventh intercostal anterior axillary line. A Lap-protector Minimini ${ }^{\circledR}$ (Hakko Medical, Tokyo, Japan) was inserted to serve as an aperture for the assistant's devices (Figure 3). A monitor with two screens 

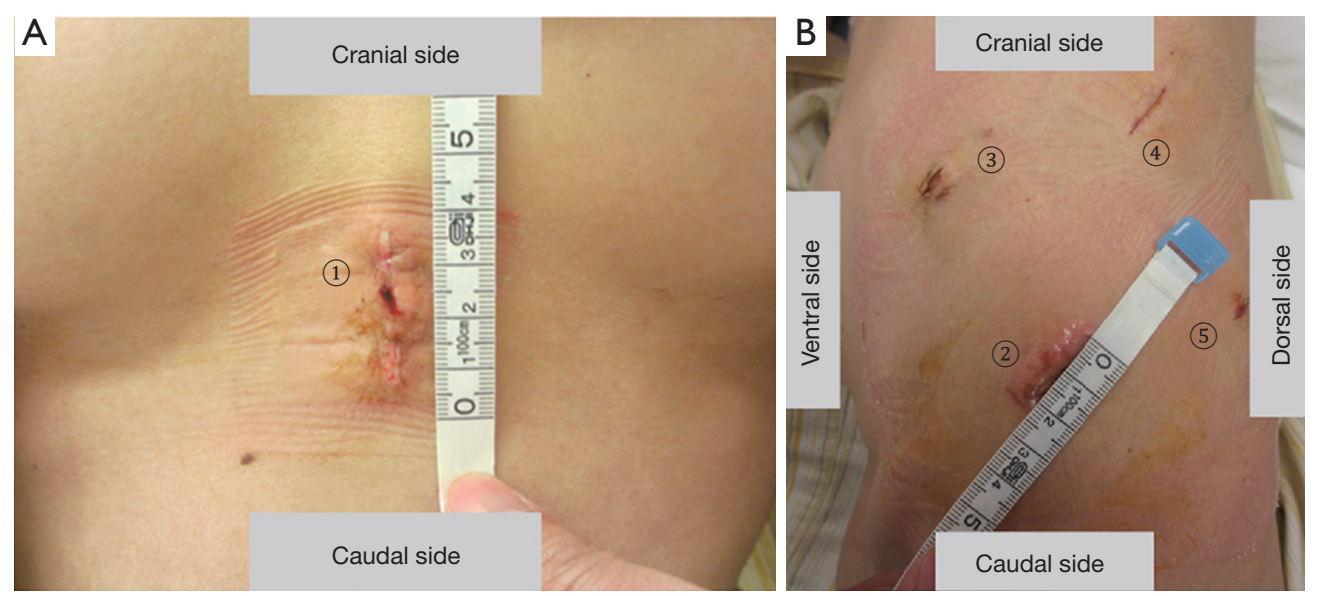

Figure 3 Position of the incision. (A) Supine position; (B) right lateral position. (1)A 3-cm skin incision in the subxiphoid area; (2)a 2-cm incision for the operator's right hand; (3) a 7-mm incision for the operator's left hand; (4) a 10-mm incision for the thoracoscope; (5) a 15-mm incision for the assistant.
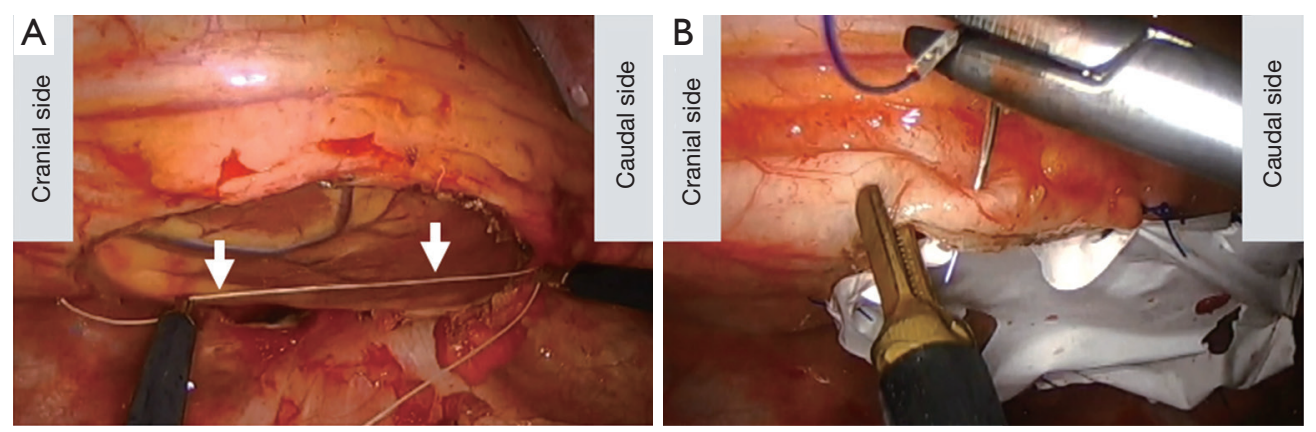

Figure 4 Intraoperative images. (A) A stretched silk thread (arrows) was used to estimate the length of the pericardial defect; (B) a needle was handled to suture the pericardium and covering material. The pericardium was flipped so that neither the tip nor the end of the needle was pointed towards the heart.

was used. One screen was rotated at the time of surgery.

When we checked the condition of tumor and thoracic cavity, we found that tumor had invaded into the pericardium. The pericardium around the tumor was resected with scissors, and a bipolar vessel sealing system was used to create a margin. Since it was confirmed that there was no dissemination in either the thoracic cavity or pericardial cavity, we decided to continuously perform tumor removal.

Similar to the procedure for conventional lingular segmentectomy, the lingular segmental veins (V4/5), arteries (A4/5), bronchi (B4/5), and segmental plane were dissected. The \#11 and \#12 lymph nodes were also dissected. There was no metastasis. After the mediastinal pleura were opened at the cardiophrenic angle, the specimen was put into a bag and removed through the subxiphoid incision.
A thread was used to estimate the size of the pericardial defect (Figure 4A). The thread was inserted with a pair of forceps and set to the defect and stretched. We grasped the thread under thoracoscopy so that the distance from the tip of the thread to grasped point was the diameter of the defect. On the basis of to the length of the thread, Gore-tex ${ }^{\circledR}$ covering material (sheet thickness $0.1 \mathrm{~mm}, \mathrm{WL}$ Gore \& Associates, Phoenix, Arizona) can be cut to the proper size. The size was $8 \mathrm{~cm}$ x $6 \mathrm{~cm}$. We made two small fenestrations in the covering material.

First, the deep side of the pericardium was handled with three threads under thoracoscopic guidance, and the corresponding side of the covering material was handled with the threads outside of the thoracic cavity. The surgical sutures used were 3-0 Prolene ${ }^{\circledR}$ RB-1 (Ethicon, Somerville, $\mathrm{NJ}$, USA). Then, the covering material was inserted into 
the thoracic cavity, and the threads were ligated. The remaining part of the pericardium and the covering material were sutured with 6 threads. When we handled the needles, we made sure that both the tip and the end of needles were not pointed towards the heart (Figure $4 B$ ). To prevent heart injury, we moved the sutured materials rather than handled the needles. A chest tube was placed in the thoracic cavity. There was no cardiac event during operation.

\section{Comments}

In our patient, thoracoscopic surgery was associated with three major problems. One was how to remove the large mass from the thoracic cavity. Because ports for videoassisted thoracoscopic surgery (VATS) are generally placed in the side-chest intercostal space, rib retraction or cutting is required to remove a mass larger than the intercostal space (3). The anterior rib cutting technique for delivering large tumors in VATS was reported by Sihoe et al. (3). They reported that the mean size of tumors removed by the technique was $5.4 \pm 3.4 \mathrm{~cm}$. Although the tumor size in our patient was slightly smaller than the reported mean, rib cutting might have been necessary to deliver the tumor through the intercostal incision because our patient was a small woman. Removing the specimen through the subxiphoid incision was one method used to resolve this problem. Kato reported that this method can expand the indications of C-VATS to include T2 and T3 tumors without rib resection and reduce postoperative pain as compared with patients who undergo rib resection (4). A technique for VATS surgery with the use of three small ports in the side-chest and one subxiphoid incision was reported by Nardini et al. and was called microlobectomy. The technique and the concept were very similar to those in our patient (5). Nardini et al. commented that this technique has two advantages. One is that the use of small instruments in the intercostal space should cause no postoperative pain and that subxiphoid retrieval avoids making an incision in the intercostal muscles and compressing the neurovascular bundles. This is exactly why we did not extend the intercostal incision, but created a subxiphoid incision. The other advantage is that the procedure performed by this approach is basically similar to that used with a normal VATS approach. Recently, procedures for complex VATS surgery via a uniportal approach have been reported, and a uniportal approach can also be one option.

The use of a uniportal subxiphoid approach may facilitate the retrieval of a large tumor without causing neurovascular bundle damage (6). However, the ability to perform different procedures from the conventional VATS procedure will require special training. Therefore, we believe that our approach is a reasonable and familiar technique that allows large tumors to be retrieved by means of a conventional VATS approach.

A technique using a uniport approach to perform extracorporeal wedge resection in the lingular segment has been reported (7). Since the lingular segment is near anterior chest wall, the peripheral lingular segment can be easily pulled out from the chest wall. However, the approach was not suitable for our patient, in whom an incision of at least 4-5 cm, similar to general anterior thoracotomy, was necessary because the tumor was large and hilar dissection was required.

The second problem was how to resect the pericardium. Since the pericardium is adjacent to the heart, care must be exercised to avoid injuring the heart. However, it is not easy to thoracoscopically resect the pericardium with the use of an electric device because three-dimensional delicate control is required. A bipolar vessel sealing system was found to be a helpful device because both blades of the bipolar vessel sealing system were covered with insulators, and the temperature in the tissue adjacent to the blades was lower with the use of a bipolar vessel sealing system than with the use of an ultrasonic scalpel and electric cautery (8). This can reduce the risk of adjacent tissue injury.

The third problem was how to reconstruct the pericardium. First, the size of the defect had to be measured under thoracoscopic guidance. We used a thread instead of a hard ruler, which is usually difficult to insert into the thoracic cavity. The length of the thread was used to estimate the size of the covering material, which should be slightly larger than the defect. This method was a very convenient and easy way for cutting covering material of the proper size. Next, the material had to be sewn onto the pericardium. Although pericardial reconstruction by robotic surgery was reported by Suda et al., the procedure requires several precautions, especially when handling the needles to perform suture safely under two-dimensional thoracoscopy (9). Since the tip and the end of the needle can injure the heart, rotation of the needles may also be dangerous. We preferred to move the sutured materials rather than the needles (10). This method can make it relatively safe to handle the needles near the heart. Moreover, we handled some needles outside of the thoracic cavity during part of the pericardial reconstruction. Migliore et al. reported a minimally invasive technique for 
extracorporeal wedge resection with a small incision (7). Even with a VATS approach, including extracorporeal handling can lead to safe and easy minimally invasive surgery.

Thoracoscopic pericardial resection and reconstruction are technically challenging. However, some of the precautions described above make the procedure feasible and less invasive.

\section{Acknowledgments}

Funding: None.

\section{Footnote}

Conflicts of Interest: All authors have completed the ICMJE uniform disclosure form (available at http://dx.doi. org/10.21037/jovs.2018.12.06). The authors have no conflicts of interest to declare.

Ethical Statement: The authors are accountable for all aspects of the work in ensuring that questions related to the accuracy or integrity of any part of the work are appropriately investigated and resolved. All procedures performed in studies involving human participants were in accordance with the ethical standards of the institutional and/or national research committee(s) and with the Helsinki Declaration (as revised in 2013). Written informed consent was obtained from the patient for publication of this manuscript and any accompanying images.

Open Access Statement: This is an Open Access article distributed in accordance with the Creative Commons Attribution-NonCommercial-NoDerivs 4.0 International License (CC BY-NC-ND 4.0), which permits the noncommercial replication and distribution of the article with the strict proviso that no changes or edits are made and the original work is properly cited (including links to both the formal publication through the relevant DOI and the license). See: https://creativecommons.org/licenses/by-nc-nd/4.0/.

doi: 10.21037/jovs.2018.12.06

Cite this article as: Nagashima T, Ito H, Samejima J, Inafuku K, Nakayama H, Masuda M. Video-assisted thoracoscopic surgery lingular segmentectomy with pericardial resection and reconstruction. J Vis Surg 2018;4:254.

\section{References}

1. Committee for Scientific Affairs, The Japanese Association for Thoracic Surgery, Masuda M, et al. Thoracic and cardiovascular surgery in Japan during 2014: Annual report by The Japanese Association for Thoracic Surgery. Gen Thorac Cardiovasc Surg 2016;64:665-97.

2. Takuya Nagashima, Hiroyuki Ito, Joji Samejima, et al. Procedure for thoracoscopic lingular segmentectomy with pericardial resection and reconstruction. Asvide 2018;5:925. Available online: http://www.asvide.com/ article/view/29142

3. Sihoe AD, Chawla S, Paul S, et al. Technique for delivering large tumors in video-assisted thoracoscopic lobectomy. Asian Cardiovasc Thorac Ann 2014;22:319-28.

4. Kato M, Onishi H, Furugaki K, et al. New Approach to Complete Video-assisted Thoracoscopic Lobectomy in T2 and T3 Non-Small Cell Lung Cancer. Anticancer Res 2015;35:3585-9.

5. Nardini M, Bilancia R, Solli P, et al. Microlobectomy: completely portal pulmonary lobectomy. J Vis Surg 2018;4:153.

6. Li L, Tian H, Yue W, et al. Subxiphoid vs intercostal single-incision video-assisted thoracoscopic surgery for spontaneous pneumothorax: A randomised controlled trial. Int J Surg 2016;30:99-103.

7. Migliore M, Criscione A, Parfrey H. A hybrid singletrocar VATS technique for extracorporeal wedge biopsy of the lingula in patients with diffuse lung disease. Updates Surg 2012;64:223-5.

8. Tirelli G, Camilot D, Bonini P, et al. Harmonic Scalpel and Electrothermal Bipolar Vessel Sealing System in Head and Neck Surgery: A Prospective Study on Tissue Heating and Histological Damage on Nerves. Ann Otol Rhinol Laryngol 2015;124:852-8.

9. Suda T. Robotic subxiphoid thymectomy. J Vis Surg 2016;2:118.

10. Horvat T, Fudulu D. Pericardial Reconstruction in Thoracic Surgery. CTSNet, 2010. Available online: https://www.ctsnet.org/article/pericardial-reconstructionthoracic-surgery 